\title{
LA PRODUCCIÓN ORAL DE NIÑOS DE PAMPLONA: CODE-SWITCHING Y CALCOS EUSKARA-CASTELLANO
}

\author{
Orreaga IBARRA Murillo \\ Universidad Pública de Navarra (España)
}

\section{RESUMEN}

Se analiza en este artículo la tipología del code-switching (CS) vasco-castellano en la producción oral de niños de 8 y 9 años. La investigación se lleva a cabo en un centro de inmersión en euskera de Pamplona, donde la docencia se imparte íntegramente en esta lengua. El análisis se basa en los datos obtenidos en el aula a través de la observación no participativa. La investigación muestra tres tipos de CS: (1) intraoracional, el más numeroso; (2) interoracional y (3) emblemático. Se concluye que el CS es una muestra de competencia comunicativa del niño, que selecciona las expresiones más adecuadas para su propósito comunicativo.

Palabras ClaVE: bilingüismo, euskera-castellano, cambio de código, contacto de lenguas.

\section{AbSTRACT}

This article deals with the typology of code-switching (henceforth CS) BasqueSpanish in the light of the spontaneous oral production of children aged between 8 and 9 in Pamplona. The research is carried out in a primary school, where the classes are completely taught in Basque. The objective of this article is to describe the CS phenomena attested in the corpus of these oral productions. The analysis is carried out using the data obtained through non-participatory observation. The research shows three types of CS: (i) intrasentential (the most frequent type), (ii) intersentential and (iii) emblematic. We conclude that the CS is a sample of communicative competence of the child, which selects the most appropriate expressions for the intended communicative purpose.

KEYWORDS: bilingualism, Basque-Spanish, code-switching, language contact. 


\section{INTRODUCCIÓN}

El objetivo de este trabajo consiste en analizar las producciones orales que presentan code switching (CS) entre euskera-castellano en el habla de los niños de 8-9 años, en una escuela de los alrededores Pamplona; en concreto en el centro Alaitz de Barañain. Para ello, aportamos algunos datos sobre la extensión y el uso del euskera en la zona de Pamplona. Es cierto que el número de hablantes vascófonos ha aumentado en Pamplona, no obstante, sigue siendo bajo, tal y como se aprecia en el siguiente gráfico: un $69,7 \%$ se desenvuelve mejor en castellano, un $24,5 \%$ en ambas igual y un $5,8 \%$ mejor en euskera.

GráFICO 1. Facilidad para hablar en zona de Pamplona

(Eusko Jaurlaritza, Gobierno Vasco 2013)

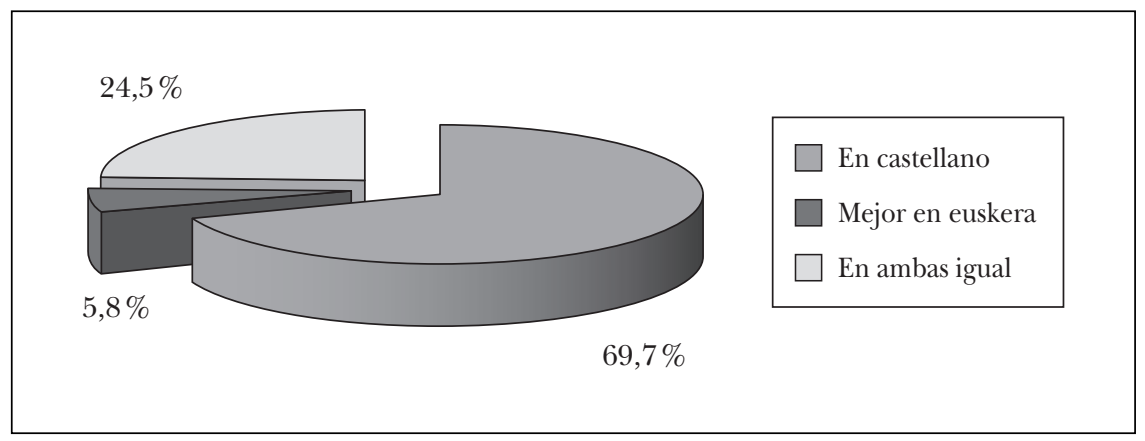

\section{Metodología}

Los datos obtenidos en el trabajo provienen de la investigación cualitativa, en concreto de la convivencia y la práctica profesional como maestro de prácticas de un alumno de Magisterio durante un mes ${ }^{1}$. La investigación cualitativa se ocupa del análisis del discurso 'natural', se opone a la investigación en contextos más artificiales, tratando de extraer significados sociales del discurso estudiado (Guest, Namcy y Mitchell 2013).

En cuanto al entorno escolar, los niños proceden de un entorno social actual con un nivel académico homogéneo y estatus económico medio. La clase se compone de 16 niños y niñas de 8 y 9 años; los padres de 9 de ellos no hablan euskera; los de 7 lo conocen en cierto grado; es decir, para

${ }^{1}$ Mi agradecimiento a Asier Aranguren por el trabajo de recopilación de datos durante su estancia en las Prácticas escolares, cuyo trabajo posteriormente dirigí. 
4 alumnos es la lengua de uso en ocasiones, y para 3 de uso cotidiano. Es la única lengua que utiliza el maestro y el personal del centro y se intenta que los niños se desenvuelvan en ella.

Nuestro análisis lo basaremos en la observación no participativa en las clases. De esta manera se evita la «paradoja del observador», en la que el investigador es el principal escollo para que los hablantes se expresen. Los niños desconocían el objetivo mientras que el profesor recopilaba los datos; las producciones de CS y calcos son espontáneas y no están contextualizadas por preguntas anteriores. Posteriormente se contabilizan los calcos, pero no son objeto de análisis en este artículo.

\section{CONSIDERACIONES TEÓRICAS}

El CS vasco-español, que es un fenómeno antiguo en nuestra comunidad, está recibiendo cada vez más atención (Lantto 2012; Muñoa 1997; Ibarra 2010, 2014) y no solo es propio de niños (Ezeizabarrena y Aéby 2010) o de jóvenes o de los hablantes que no han adquirido completamente la lengua. Por el contrario, está presente en todos los hablantes bilingües, tanto en las comunidades de hablantes nativos vascoespañoles (Ezeizabarrena y Manterola 2004; Etxebarria 2004) como entre hablantes vascofranceses (Epelde y Oiartzabal 2009). Como sucede en el contacto de otras lenguas, y más si cabe, en este caso de convivencia de dos lenguas en situación diglósica, (Cenoz 2008), la alternancia lingüística ha sido rechazada como propia de los hablantes no completos o se ve como una degradación del lenguaje. Por otra parte, el CS ha sido un fenómeno ignorado y poco estudiado; en parte, por la situación de diglosia en la que se encuentra la lengua vasca con respecto a la española. Scotton (1993: 48), sin ir más lejos, relata cómo durante años de investigación lingüística en África, ignoró el CS como fenómeno lingüístico.

Appel y Muysken (1996) consideran cambio de código o code switching tanto a las inserciones de un elemento como a las que ocurren con más componentes, todas forman parte del habla del bilingüe. Se trata de una manifestación lingüística que se realiza sin esfuerzo y se difunde muy rápidamente. Es conocida la clasificación de la alternancia que proponen estos autores y que nosotros también la aplicaremos para clasificar las producciones:

a) alternancia interoracional;

b) alternancia intraoracional, y

c) coletillas o alternancia emblemática.

Fantini (1992) resume de esta manera las motivaciones por las que el hablante bilingüe puede alternar la lengua con diferentes propósitos: 
a) por efectos pragmáticos: usando la lengua que está marcada o que no es la esperable, para enfatizar algo;

b) como un recurso metalingüístico: para verlos desde otro punto de vista lingüístico;

c) en respuesta a los tópicos; en relación a las experiencias en una lengua (contraste cultural);

d) como triggering, es decir, como una palabra que parece que es de las dos lenguas y actúa como trigger; que lleva al hablante a olvidar en qué lenguaje está hablando, y continúa en la otra lengua;

e) por el contexto de comunicación, cuando el contexto o la experiencia está conectado a otra lengua (contraste cultural);

f) para evitar problemas de traducción;

g) como notas para evitar un vocabulario desconocido;

h) como notas para una función metafórica o pragmática.

Este mismo autor explica que el bilingüismo implica no solo las lenguas a usar sino también el conocimiento de ambas lenguas, así como las habilidades comunicativas del hablante y la consciencia de las mismas.

En este sentido, Lam (2001: 93) define el bilingüismo como «el fenómeno de competencia y comunicación en dos lenguas». En sentido más estricto, en cuanto a las ventajas y desventajas del mismo, una creencia extendida sobre el bilingüismo se refiere a las ventajas cognitivas y/o la confusión lingüística de estos hablantes. Hace algunas décadas, se creía que los bilingües tenían desventajas educativas y socioculturales (ej. niños mexicanos en EE.UU.). Sin embargo, estudios posteriores (Romaine 1999) han demostrado que la inteligencia verbal de los bilingües es, aunque se desarrolla de manera tardía, mayor que la de los niños monolingües.

Por otra parte, las actitudes, el grado de dominio de la lengua, el tipo de lengua usada, y la interacción de las mismas son factores que intervienen en la producción del bilingüe. Etxebarria (1995: 170) diferencia entre code-switching, code-mixing y préstamos. Respecto al (CS) dice: «el hecho de utilizar uno o más elementos de la lengua $\mathrm{B}$ en un enunciado de la lengua A, o de alternar entre A y B en el mismo enunciado».

Para que se produzca el CS tienen que cumplirse generalmente dos condiciones. Como indicó Poplack (1980), en la primera regla, la del morfema independiente: no se puede sufijar un elemento gramatical y uno derivado de L2 a L1, porque la alternancia está prohibida en este tipo de unidades. La segunda regla, la de equivalencia estructural, establece que hay que mantener el orden de los dos constituyentes y no se puede dar la sustitución si no tienen los constituyentes el mismo orden. Es decir, el cambio no puede violar las reglas sintácticas de ninguna. De tal forma que el orden de los elementos que preceden y suceden al cambio ha de ser gramatical en ambas lenguas. 
En cuanto al proceso de sustitución de una lengua por otra, Poplack (1980) señala que la alternancia de lenguas exige que los patrones gramaticales de ambas lenguas no se vean alterados. Es decir, que el hablante no puede sustituir en cualquier punto de la frase, porque hay ciertas restricciones. También considera que el CS es producto de la competencia bilingüe del hablante, no un fallo de la competencia del mismo, sino una consecuencia de la completa capacidad del bilingüe para alternar entre estas estructuras. Por otra parte, este fenómeno depende del interlocutor, de la formalidad de la situación y de la situación comunicativa: «Solo aquellos hablantes que han aprendido ambas lenguas a una edad temprana alcanzarán el nivel de competencia preciso para emplear las dos lenguas en la misma oración» (Appel y Muysken 1996: 180).

Insistiendo en esta idea, para algunos investigadores (Mondada 2007) esta llega a ser una manera de hablar no marcada y habitual. En este sentido, los investigadores encontraron que «lejos de las creencias superficiales de profanos que ven el fenómeno como una clara manifestación de corrupción y deterioro lingüísticos, de conducta verbal indeterminada, el cambio de código resulta gobernado por requisitos funcionales y pragmáticos» (López Morales 1989/1994: 173).

Gumperz (1982), desde el punto de vista funcional, afirma que el CS aporta información. No obstante, esto no supone que a la alternancia de código se le asigne siempre una información precisa. Gumperz sugiere que el hablante bilingüe de una comunidad de habla elige el CS como una opción comunicativa propia; es decir, desde el punto de vista lingüístico el CS constituye un modo de discurso. De la misma manera, se puede decir que la alternancia de estilo o de dialecto es una opción del hablante monolingüe. La alternancia serviría para una función expresiva y esta posee, a su vez, una función pragmática. Finalmente, el autor sostiene que el cambio de código se encuentra más frecuentemente en el habla informal.

A juicio de Betti (2010: 32), en las comunidades de hispanohablantes con el spanglish, «el uso de ambas lenguas es decir, la alternancia de estos dos códigos no se puede considerar un signo de deterioro. En un ambiente bilingüe cualquier oyente atento puede darse cuenta de que los protagonistas de una interacción dialógica pasan de un idioma a otro dando vida así a una forma rentable de comunicación». Kim (2006: 51) afirma que el CS es «a linguistic tool and a sign of the participants' awareness of alternative communicative conventions».

Por otra parte, en el análisis realizado por Ezeizabarrena y Aéby (2010: 76), con bilingües vasco-españoles de 0 a 6 años, se afirma que hay una frecuencia muy baja de este fenómeno en la producción de los niños bilingües que adquieren dos lenguas a edades precoces. Los niños no muestran una dirección clara de CS y durante estos primeros años desarrollan las 
dos gramáticas paralelamente y sin interferencias a los niveles estudiados. Quizás la distancia tipológica entre las dos lenguas consideradas puede jugar un papel importante desde este punto de vista. Desmiente, por tanto la hipótesis de fusión gramatical en las primeras etapas de la producción lingüística. Por otra parte, les permite observar una gran frecuencia de CS de tipo extrasintagmático más que de tipo intrasintagmático. Esto indica que la elección de la lengua en las producciones de CS responde a una motivación o dominancia pragmática. Desde el punto de vista cuantitativo la frecuencia de este fenómeno de alternancia en la frase es extremadamente baja en las producciones de niños bilingües que adquieren las dos lenguas a edades precoces $(<4 \%)$.

Finalmente, el CS vasco-español es un rasgo convencional, aceptado en la comunicación bilingüe entre los jóvenes. Hay que tener en cuenta que la conversación coloquial es un ámbito donde el discurso no está guiado ni monitorizado. El CS forma parte habitual del repertorio de los hablantes jóvenes bilingües vascos (cf. Ibarra 2010, 2011), aunque los profesores corrigen estas manifestaciones, debido a que se ve como un acto de habla impuro y una falta de atención y de peligro potencial de la pureza de la lengua. Así, tal y como afirman Bhatia y Ritchie (2004: 350), «the vast majority of bilinguals themselves hold a negative view of code-mixed speech They consider CS to be a sign of laziness».

A continuación, analizaremos el fenómeno de CS en los corpus de estas producciones orales. Asimismo, discutiremos la naturaleza del modelo, relativo a la tipología de los elementos trasferidos y la dirección de estas transferencias. El code-switching ha sido definido como un rasgo de incompetencia en los niños bilingües. Sin embargo, nuestra tesis en este artículo es mostrar que el CS es un comportamiento unido al entorno bilingüe y que no solo la ejercen los bilingües adultos, sino también los niños como una efectiva estrategia comunicativa.

\section{Cambio de código emblemático}

En este tipo de inserciones se incluyen exclamaciones, coletillas o paréntesis, y conectores de una lengua diferente de la del resto de la oración. Estos conectores invariables funcionan como emblemas del carácter bilingüe de una oración completamente bilingüe. En nuestro caso, están compuestos por preposiciones, adverbios, locuciones adverbiales. Enríquez (2017: 192). estudia el uso de los conectores pues, pero, porque en el habla infantil castellana y concluye que aparecen tempranamente en el lenguaje infantil, entre los 2 y los 4 años. Estos enlaces actúan como marcadores puesto que manifiestan funciones y valores de tipo comunicativo. «Las conjunciones 
del castellano, con excepción del si, cumplen primero funciones del tipo pragmático». A modo de ejemplo:

(1) Pero, zein da hori?

'Pero, quién es ese'

(2) Hodei, al menos sakatu koadernoa!

'Hodei, al menos saca el cuaderno'

(3) Pues entonces da Irunerena!

'Pues entonces es de Irune'

(4) Por si acaso eman liburua

'Por si acaso dame el libro'

En la conversación adulta, por el contrario, las inserciones emblemáticas se utilizan cuando se trata de enfatizar un elemento de la conversación con frases o expresiones ya hechas (Muñoa 1997). Se trata de fórmulas que subrayan la expresividad y tienen por objetivo enfatizar utilizando expresiones ya marcadas y esta es la razón posible de que el hablante joven alterne con el castellano (Ibarra 2014).

Sin embargo, en los casos analizados de habla infantil la alternancia de muletillas no supone una alternancia con objeto de enfatizar lo anteriormente dicho, sino que son conectores, en castellano están presentes cuando el niño está inmerso en un contexto narrativo o en una disputa verbal (Enríquez 2017: 194). Estos fragmentos de discursos infantiles no se suceden como turnos en una interacción y no presenta una dinámica de conversación donde hay un hablante oyente y en la que la relación entre ellos es simultánea (Briz 1998). En realidad, estos ejemplos no constituyen un tipo de conversación prototípica, porque los niños, cuando elaboran el mensaje, no tienen demasiado en cuenta lo que ha dicho el interlocutor que lo precede.

\section{Alternancia intraoracional}

Este tipo de alternancia, que se da dentro de la oración, supone conocer las estructuras gramaticales de las dos lenguas. Por tanto, presupone una de las condiciones que es la de la equivalencia. De esta manera, el orden de la frase tiene que ser gramatical; es decir, tiene que coincidir el orden y la gramática y el cambio se realizaría dentro de la misma frase. Lingüistas como Poplack (1980) señalan que este es el cambio más complejo pues requiere que el hablante controle los dos sistemas lingüísticos a la vez.

Las hemos clasificado en: inserciones puras, adaptaciones de vocabulario, inserciones con preposiciones y, finalmente, las construcciones con si..., que... 


\subsection{Inserciones puras}

En este apartado incluimos los lexemas que se insertan sin ninguna marca morfológica; de manera pura se trata de inserciones en el nivel de núcleo o inserciones de islas. Lo denominamos CS, porque no son préstamos integrados. En este sentido, Mendieta (1999: 11) sobre el préstamo y el cambio de código, destaca: «Se consideran préstamos las palabras que se realizan formalmente siguiendo las reglas que se aplican a las palabras patrimoniales de la misma categoría. Por el contrario, las palabras del cambio de código no presentan una realización formal común con el resto de los miembros de la misma categoría...». Y agrega: «La realización formal comprende tanto la adaptación fonética como la adaptación morfológica y sintáctica de la palabra o el grupo».

Por otra parte, en cuanto al tipo de préstamo es difícil establecer jerarquías de préstamos por medio del simple recuento de elementos de un corpus; sin embargo, diversos investigadores las han elaborado (cf. Appel y Muysken 1996: 254) y demuestran que los sustantivos se prestan con mayor frecuencia que los verbos, los verbos más fácilmente que los adjetivos, etc. En general, existe consenso en que los sustantivos son los elementos que se prestan más fácilmente.

En nuestro caso, encontramos sustantivos, inserciones puras, seguidos del determinante en euskera bat 'un, uno':

(5) Nire amaren tío bat du huerta bat (falta de ergativo)

'Un tío de mi madre tiene una huerta'

(6) Nor echatu da pedo bat, es que sonatu du

'Quien se ha echado un pedo, es que ha sonado'

(7) Atzo erori nintzen eta egin nuen moratón bat eta orain dolitzen zait

'Ayer me caí y me hice un moratón y ahora me duele'

(8) Beste egunean sartu zen abeja bat

'El otro día entró una abeja'

(9) Egun batean egon zen incendio bat nire herrian

'Un día hubo un incendio en mi pueblo'

Hay que precisar que las estructuras mixtas vasco-españolas a veces contienen un determinante español; este es específico desde el punto de vista del género, mientras que en el euskera el determinante no lleva más que las marcas del nombre y del caso.

La mayor parte de las veces los sustantivos insertos tienen la función de complemento directo y hay una tendencia a que la inserción aparezca pospuesta al verbo: 
(10) Asier dauka parecido.

'Asier tiene parecido'

(11) Nik gaur ez, zergatik dut foto de comunión.

'Yo hoy no porque tengo foto de comunión'

(12) Nuen razón

'Tenía razón'

En algunos casos, como el de 'foto de comunión' (11), creemos que se trata de un caso de sintagmas marcados, como sucede con los nombres de calles o expresiones hechas o sintagmas ya prefijados (cf. Epelde y Ohiarzabal 2009). También ocurre con el ejemplo ‘de regalo' (13) cuya inserción es más probable se tome de la L1, porque el contexto y la experiencia está conectada con el castellano.

\subsection{Inserciones preposicionales}

Este tipo de alternancia también es propia de hablantes adultos que alternan vasco/francés según han investigado Epelde y Oiartzabal (2009). Es un tipo de alternancia pura, ya que la segunda parte se introduce a manera de coletilla y está introducida por una preposición. Es muy habitual dentro de las estructuras oracionales y requiere cierto dominio para no cambiar en puntos no permitidos por ambos sistemas lingüísticos. Estas son las preposiciones (contra, con, en, al, de, por, para) que preceden a los sintagmas preposicionales (cf. Ibarra 2014). Cuando ocurre el CS, frecuentemente el cambio de lengua afecta a todo el sintagma, y por tanto, todo el sintagma preposicional estará en L2.

(13) Hau compratu behar duzu tienda batean eta de regalo eramaten duzu beste bat

'Esto lo tienes que comprar en una tienda y de regalo te llevas otro'

En (14), vemos que hay una inserción de sintagmas preposicionales en L2, con preposiciones para, en. En estos casos las inserciones están pospuestas al verbo y aparecen al final de la oración. Es preciso señalar que en euskera estos sintagmas pueden ocupar un lugar libre en la frase (pueden estar antepuestos o pospuestos al verbo). En este primer caso se trata de una locución adverbial entera:

(14) Hau uzten dugu para luego

'Esto lo dejamos para luego'

También puede ser un sintagma nominal: 
(15) Eske begiratzen tanto rato baloiari mareatzen naiz 'Es que mirando tanto rato al balón me mareo'

En el siguiente caso, pensamos que al niño le faltaba la palabra furgoneta, y salta al castellano con los determinantes y preposiciones necesarias, de manera que esta palabra hace de triggering y atrae al resto del sintagma. Clyne (1972: 24) clasifica cinco tipos de palabras que pueden actuar como trigger. Se trata de algunas palabras 'neutrales' que podrían pertenecer a cualquier lengua, las cuales causan al hablante «que pierdan la orientación» y continúen su discurso en una lengua diferente de la que ha empezado.

La tendencia a insertar el sintagma completo en castellano está documentada (Epelde y Oiartzabal 2009), aunque resulta un tanto extraña, ya que el euskera es una lengua aglutinante y las inserciones lexicales suelen realizarse fácilmente en este tipo de lenguas (Muysken 2000: 76):

(16) Konbertitzen naiz en una furgoneta

'Me convierto en una furgoneta'

A veces hay producciones en las que solo un lexema hiztegi 'diccionario' está en esta lengua; habría que interpretarlo como CS de castellano a euskera.

(17) ¿Puedo mirar en el hiztegi una cosa?

Este tipo de inserciones (17) son habituales porque forman parte de un caudal lexical que se usa con asiduidad en el entorno de ikastolas: la txamarra 'chaqueta', la andereño 'la señorita', la gela 'el aula', los etxekolanas 'las tareas', el hiztegi 'diccionario', un marrazki 'un dibujo'. Algunos de ellos son préstamos ya integrados como los dos primeros. En el habla de los adultos también existen las inserciones de euskera en discurso en francés y castellano, aunque siempre son menos numerosas (cf. Epelde y Ohiarzabal 2009).

\subsection{Inserciones lexicales adaptadas a nivel de núcleo}

Tal y como hemos apuntado, existen sustantivos que se toman prestados del castellano y a los que se les añaden sufijos; esta alternancia es típica de lenguas aglutinantes: «A case affix from one language is attached to the nominal constituent from another one, are typical of code-mixing involving agglutinative languages such as Basque, Qechua...» (Muysken 2000: 76). En el caso del euskera se añade los siguientes desinencias en euskera: $-n$ de inesivo (misa-n, camioneta-n ej. 18, 24), -a, determinante singular (ejercicio-a, diccionario-a, panadero-a ej. 19, 23, 24), -rekin morfema de compañía, 
(carne-rekin, fiebre-rekin) $(20,21)$, finalmente, -ago morfema graduador (lento-ago) (22).

(18) Es que du comunión eta horregatik ikusiko dut misan

'Es que tiene comunión y por eso lo veré en misa'

(19) Horrela egiten dugula ejercicioa

'Que así hacemos el ejercicio'

(20) Larunbatean jan nuen patata frijituak carnerekin

'El sábado comí patatas fritas con carne'

(21) Nago fiebrerekin, es que dut asko beroa

'Estoy con fiebre, es que tengo mucho calor'

(22) «Bat» esan nahi du lentoago joan behar zarela

'«Uno» quiere decir que tienes que ir más lento'

(23) Ahal da hartu diccionarioa?

'¿Se puede coger el diccionario?'

(24) Nire herrian dago bar bat eta panaderoa etortzen da camionetan

'En mi pueblo hay un bar y el panadero viene en camioneta'

Este tipo de inserciones no consolidadas son a nivel de núcleo, tanto que en las inserciones vascas no siempre es fácil saber si se trata de formas CS o de préstamos; el límite entre ambos es impreciso en el caso de préstamos no consolidados.

\subsection{Inserción de verbos}

Analizamos la inserción de palabras con una adaptación morfológica o fonológica en la lengua en la que se inserta. Se denomina también alternancia de código morfofonológica y se trata de la alternancia de sonidos para adaptarse de la L1 a L2. Siguen una estructura semejantes al CS ingléscastellano: mapear, lunchear, resetear, hackear... En general predominan los verbos; como se sabe, muchos de los verbos actuales del euskera proceden del castellano y se han lexicalizado desde hace mucho tiempo, añadiendo el morfema - $d u$ o - $t u$. Algunos autores (Fernández Ulloa 2004) los consideran como un caso aparte, dada la dificultad de determinar si se trata de CS o de préstamo.

En estos casos, la adaptación morfológica consiste en eliminar la -r final del infinitivo y añadir al verbo español el morfema de participio -tu: sudar > suda-tu, dormir > dormi-tu. Una vez construido el participio, se añade a la raíz verbal los morfemas -tzen, -tzeko, -tzera en el caso de nominaliza- 
ciones y en el de futuro se añade $-k o$, y de manera analógica se completan todas los demás inflexiones. Resulta un recurso muy productivo como lo vemos en los siguientes ejemplos extraídos de realizaciones más largas:

atropellatu 'atropellar', ari naiz buskatzen 'estoy buscando', dormitu zait 'se me ha dormido', echatu da 'se ha echado', elejitu dut 'he elegido', enamoratzen 'se enamora', enfriatuko zara 'te enfriarás', explikatu 'explicar', ez dut pegatu behar 'no voy a pegar', inbentatu behar 'hay que inventar', juntatzeko 'para juntar', kazatu nuen 'cacé', konpratu behar duzu 'tienes que comprar', kreitzen dut 'creo', llevatzen da 'lleva', mejoratu 'mejorar', nago peditzen 'estoy pidiendo', nezesitatzen dut 'necesito', nombratzen da 'se nombra', parezitzen dizu/du 'te parece', robatu didazu 'me has robado', sudatuta 'sudado/a', utilizatu behar 'hay que utilizar'.

Evidentemente, estos verbos recopilados no están integrados en la lengua vasca. No obstante, hay que señalar que esta ha sido y es la manera más habitual de crear neologismos y tecnicismos con verbos del castellano que ya se han lexicalizado: ej. funcionar > funtzionatu, explicar > esplikatu. No se puede olvidar que muchos fenómenos que inicialmente son considerados como interferencias se asientan en la lengua y pasan a convertirse en «préstamos» $\mathrm{y}$ «calcos». Estos verbos pueden sufrir un proceso de gramaticalización y desarrollarse como una marca de integración. En este sentido, la integración de los verbos ha sido estudiada por Matras (2009: 176), que explica cuatro tipos de integración verbal. Añade que hay evidencias que sugieren que las marcas inflexionales prestadas pueden también desarrollarse como marcas fonológicas integradas. Asimismo, en la comunidad vasco-francesa, los verbos del francés utilizados por los bilingües adultos son numerosos y se han adaptado al euskera con suma facilidad, según la investigadora (Epelde 2014): [exprim-atu] (fr. exprimer), [anonç-atu] (fr. anoncer), [insist-atu] (fr. insister), [nomm-atu] (fr. nommer).

Por otra parte, Muysken y Ali Kha (2014) muestran que la estrategia utilizada para la adaptación de verbos del pastún al inglés es similar a la del euskera; asimismo señalan que en situaciones de contacto lingüístico, la mezcla de códigos es una fuerza de indigenización de verbos de la lengua donante a la lengua receptora y subrayan la dificultad de determinar si una palabra es Code mixing o se trata de un préstamo.

\subsection{Construcciones con si..., que, es que...}

Si introduce una construcción independiente cuya principal función es remitir a lo inapropiado de una intervención previa, es decir, manifestar desacuerdo o incredulidad. Esta tendencia está también muy presente 
en el habla infantil y también juvenil (cf. Ibarra 2014) y marca el inicio de la frase que tras ella alterna al euskera $(25,26)$. En $(27)$, el si... es un marcador reforzado por el marcador opositivo 'tampoco', que da énfasis al enunciado.

(25) Ay, si naiz arduraduna!

'Ay! Si soy la responsable?'

(26) Ay, si nago sekatzen

'Ay, si me estoy secando'

(27) Si tampoco ez dira kontatu behar

'Si tampoco hay que contarlas'

Enríquez (2017: 165) estudia la aparición y el uso de este enlace en el habla infantil. Diferencia dos funciones, como conector sintáctico y como conector pragmático, y añade: «La mayor parte de los niños empiezan a usar este conector a partir de los 3 años». Asimismo, señala que las particularidades gramaticales y pragmáticas todavía no se tienen claras en la gramática adulta, donde frecuentemente ha sido analizado partiendo de modelos escritos de estructuras condicionales, dejando desatendida su utilización en el registro coloquial y oral de la lengua (Enríquez 2017: 172). En los casos analizados hace función de conector pragmático y no responde a una estructura condicional.

Por otra parte, aparecen oraciones que empiezan con que y funcionan a manera de aviso, para hacer una consideración.

(28) Que ez dakit zein da! 'Que no sé quién es'

(29) Que kostatu zaigu rato asko egitea e! 'Que nos ha costado mucho hacer eh!'

En estas circunstancias que actúa como conector y participa en la formación de una construcción iniciada con que, a manera de advertencia. Es decir, introduce una causa a nivel pragmático, donde la conexión ya no se establece entre proposiciones enunciado, sino entre unidades comunicativas diferentes, entre intervenciones y enunciados distintos. En otros casos se inicia con la interjección a que, a manera de apuesta o reto:

(30) Konfunditu naiz, baino a que parecitzen du egiazkoa 'Me he confundido, pero a que parece de verdad'

De la misma manera, las iniciadas con es que son muy habituales en el lenguaje juvenil y también en el infantil. El adverbio es que se usa para dar un giro coloquial o una razón y añadir una explicación sobre algo. Es decir, 
se emplea para dar entrada a excusas, justificaciones o explicaciones. La focalización con es que y dislocación a la izquierda es un rasgo específico del estilo oral castellano. «El proscribir la manera con es que en el lenguaje escrito es un hecho histórico basado en criterios estilísticos» (Zimmermann 2002: 150).

En otros casos es que aparece como introductora de la frase, pero, posteriormente se vuelve al discurso en euskera otra vez. La construcción es que, escrita eske y sk en lenguaje escrito informal de los jóvenes (chats, whatsapps) se encuentra semilexicalizada y se puede considerar como una construcción formalmente fijada:

(31) Nago fiebrerekin, es que dut asko beroa

'Estoy con fiebre, es que tengo mucho calor'

A modo de conclusión, podemos decir que los conectores si y que de la lengua base son muy productivos, introducen la oración y posteriormente, se da el salto a la L2. Estos marcadores inalterables son habituales, ocurren espontáneamente en el habla infantil y responden a un hábito y constituyen una señal de discurso mezclado. Recuerdan los recopilados por Romaine (1989: 112), como «you know», «I mean»o «like» insertos en el lenguaje adulto español en contacto con inglés.

\section{Alternancia interoracional}

Esta alternancia respeta la gramática subyacente de cada lengua, incluso las reglas morfosintácticas de cada una de ellas; no está ligada con el cambio de contexto o de tema de conversación, al contrario, se insertan en frases condicionales y completivas, y no es toda la frase la que se inserta, sino solo los conectores que la inician.

Un tipo de alternancia interoracional es la que se inicia con la conjunción si. En castellano puede utilizarse con función pragmática o con una función sintáctica. En este caso, la partícula si actúa como conector sintáctico, sobre todo en construcciones condicionales, la construcción verbal que continúa está en presente. Responde a una estructura de modelo prototípico de condiciones reales, con las formas verbales codificadas en modo indicativo, y en presente y con el miembro que tiene el enlace antepuesto al verbo principal. En euskera la partícula $b a$ 'si' precede al verbo auxiliar, es decir, aparece postpuesta. Introduciendo el si en castellano, lo que se hace es adelantar la cláusula condicional.

(32) Baina, si zu debolbitzen diozu geratzen zara peor

'Pero, si tú lo devuelves, te quedas peor'

Trad. lit. 'Baina, zuk itzultzen badiozu, okerrago garatzen zara' 


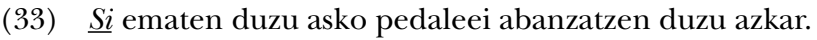

'Si le das mucho a los pedales, avanzas rápido'

Trad. lit. zuk ematen badiezu pedalei, aurreratzen duzu asko'

En (32), además de la partícula si, aparece con otro conector, que es baina 'pero', y se utiliza en contextos negativos. El resto de la cláusula está en L2.

Por otra parte, encontramos inserciones de oraciones completivas en las que la conjunción que es la que introduce la cláusula y a veces es el único elemento en castellano. En (34), incluso el inicio en L1 está en castellano y posteriormente se da el salto al euskera. En euskera se requiere un morfema completivo - la que se coloca postpuesto al verbo auxiliar. En estas realizaciones que -que es el correlato del morfema -la-, aparece antepuesto.

(34) Creo que dut gaizki, baino bueno...

'Creo que tengo mal, pero bueno...

Lit. Uste dut gaizki dudala, baina, bueno

(35) Ni (sic) ikusi dut periodikoan que galdu da abioi bat 'He visto en el periódico que se ha perdido un avión'

Lit. Ikusi dut egunkarian, galdu dela hegazkin bat

Estos casos de CS, revelan una estructura paralela a la de la lengua de origen. Además, es preciso subrayar un rasgo, la ausencia del morfema de ergativo $(36,37)$. En las realizaciones recopiladas observamos que falta el morfema de ergativo. Es decir, en euskera el sujeto de las oraciones transitivas se caracteriza por llevar el morfema $(-\mathrm{k})$, de ergativo. Este rasgo no aparece como marca en estas oraciones:

(36) Baina si $\underline{\text { zu}}$ debolbitzen diozu geratzen zara peor

'Pero si tú le devuelves, te quedas peor'

Lit. $\underline{\text { Zuk }}$

(37) Nire amaren tio bat du huerta bat

'Un tío de mi madre tiene una huerta'

Lit. Osaba batek

Este tipo de ergatividad escindida ocurre con asiduidad en el discurso bilingüe, especialmente entre jóvenes (cf. Ibarra 2011; Epelde 2014). Para los hablantes de una lengua nominativo-acusativa, como es el castellano, la inserción de la marca de ergativo presenta dificultades de aprendizaje, perfectamente comprobable en la práctica docente diaria. Es probable que la distancia tipológica entre estas dos lenguas sea la causa de la dificultad de insertar este morfema. 


\section{DISCUSIÓN Y CONCLUSIÓN}

El CS entre euskera/castellano entre bilingües adultos y jóvenes, que ha sido objeto de análisis en otros estudios (Ibarra 2010, 2014; Etxeberria 2004), puede ser interpretado como un fenómeno creativo que no implica necesariamente limitación en el uso de cada lengua, sino la posibilidad de mezclarlas por razones afectivas y expresivas. Es decir, el CS tiene que ver más con el cambio y con las variaciones estilísticas. Es una manera en la que el bilingüe introduce informalidad y se siente miembro de un grupo. Investigaciones sobre el euskera informal hablado en Bilbao (cf. Lantto 2012) muestran que los rasgos de este registro coloquial se basan en la combinación de dos elementos: el CS y el uso de los términos coloquiales españoles. También Muñoa (1997: 534) estudia las funciones pragmáticas que el CS hablado en San Sebastián desempeña; a juicio suyo, el CS es debido, en parte, a la falta de jerga en el habla urbana; a su juicio, una carencia del euskera.

En los casos analizados, el CS es una muestra de competencia comunicativa del niño, que muestra la habilidad para producir y seleccionar, del total de las expresiones correctas gramaticalmente, las que más adecuadamente gobiernan los comportamientos. Wang y Hyun (2009) apoyan esta idea que manifiesta la competencia comunicativa de los niños bilingües; así, los niños hacen uso de esta necesidad comunicativa cuando la necesitan. Por ejemplo, en algunos casos, el CS es debido a que existen sintagmas ya marcados en la L1 o a problemas de traducción para evitar vocabulario desconocido, en otros es un caso de triggering. En este sentido, el CS debería aprovecharse en la escuela para incrementar la competencia comunicativa.

En cuanto a resultados numéricos, se han recopilado 76 realizaciones en total, de ellas 4 corresponden a CS emblemático, un 5,26\%. Por otra parte, hay 57 casos de CS intraoracional, un $75 \%$. En tercer lugar tenemos 6 casos de CS interoracional, un 7,9\% y, finalmente, tenemos 9 casos de calcos lingüísticos, un $11,84 \%$.

Por una parte, concluimos que el número de casos de CS es más alto que el de calcos, como era de esperar; en parte, porque no hay efecto de observador externo y los alumnos no están pendientes de las correcciones del adulto. Se contrasta con trabajos similares donde sí existe este efecto y en ellos se encuentra mayor cantidad de transferencias lingüísticas que de CS (cf. Martínez y Sanaphre 2017: 99). Es decir, que a pesar de que el CS es muy identificable, ya que es una palabra insertada en otra lengua, su predominio, frente a los calcos, muestra que la expresión de los niños es espontánea y natural.

Por otra parte, en los casos de CS intraoracional -mucho más numeroso- es reseñable el alto número de adaptaciones de verbos, resultando ser 
GRÁFICO 2. Clasificación de las producciones

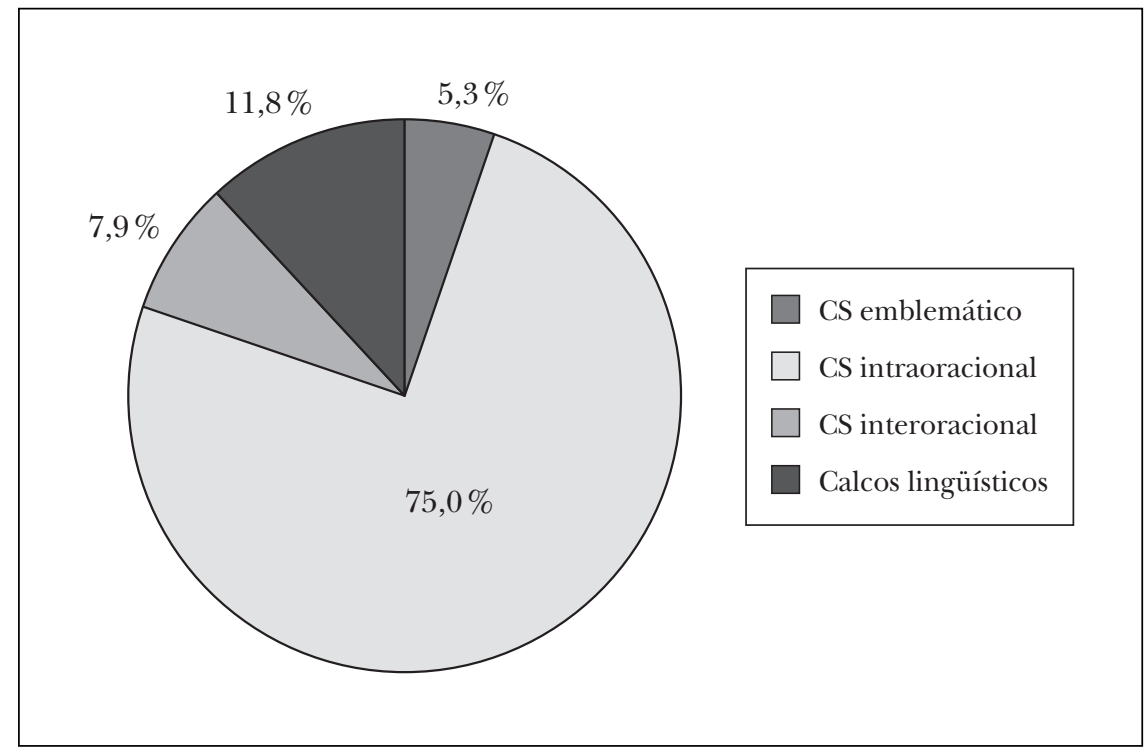

este un recurso muy productivo, la alternancia se hace de una manera no marcada. Se podría hablar de una alternancia de oraciones convencional, ya que representa un hábito al hablar entre los niños de esta edad. Por otra parte, el CS no se realiza de un modo desordenado, sino que existen una serie de patrones lingüísticos, verbos insertados, enlaces como si, que, es que, que se utilizan como inicio de las producciones, para después insertar la lengua matriz. Finalmente, existen pocos casos de alternancia interoracional que inician oraciones completivas y condicionales. 


\section{BIBLIOGRAFÍA}

Ali Khan, Arshad y Peter Muysken (2014): «Strategies for incorporating nouns and verbs in code-mixing. The case of Pashto-English bilingual speech», $L a$ purdum XVIII, 97-137.

APPel, René y Peter MuYsken (1996): Bilingüismo y contacto de lenguas, Barcelona: Ariel Lingüística.

BEтTI, Silvia (2010): «Spanglish: ¿pseudolengua o identidad?», Estudios de Lingüistica Aplicada año 28, n. ${ }^{\circ}$ 52, diciembre de 2010, 29-54.

Bhatia, Tej y C. Ritchie William (2004): «Social and Psychological Factors in Language Mixing». En William C. Ritchie y Tej K. Bhatia (eds.) (2012), Handbook of bilingualism, Blackwell Publishing, 336-352.

BRIZ, Antonio (1998): El español coloquial en la conversación. Esbozo de pragmagramática, Barcelona: Ariel Lingüística.

Clyne, Michael (1967): Transference and Triggering: Observations on the Language Assimilation of Postwar German-speaking Migrants in Australia, La Haya: Nijhoff.

ENRÍQUEZ, Iván (2017): Del discurso a la gramática en el habla infantil, Madrid: Arco/Libros.

Epelde, Iranzu y Beñat Oiartzabal (2009): «Code Switching en las variedades orientales del vasco», Baiona: IKER-UMR 5478 Basque Text and Language Study Center. HAL Id: artxibo-00389939. <https://artxiker.ccsd.cnrs.fr/artxibo00389939>.

EPELDE, Iranzu (2014): Euskal Hitzez. <https://31 eskutik.com/2014/10/06/euskalhitzez/>.

ETXEBARRIA, Maitena (1995): «El método de la investigación sociolingüística», Letras de Deusto 25, n. ${ }^{\circ}$ 68, 169-182.

- (2004): Español y euskera en contacto: resultados lingüisticos. RILI II, 2/4, 131-145.

Eusko JaUrlaritza (2013): Encuesta sociolingüistica, 2011. Vitoria-Gasteiz: Eusko Jaurlaritzaren Argitalpen Zerbitzu Nagusia.

Ezeizabarrena, Maria José y Sandrine AÉBY (2010): «Les phénomènes de codeswitching dans les conversations adulte-enfant(s) en Basque-Espagnol: une approche syntaxique», Corpus 9 (Michèle Olivieri [ed.], La Syntaxe de Corpus), 53-80. <http://corpus.revues.org/1960?\&id=1960\&file=1>.

FANTINI, Alvin (1992): Exploring bilingual behavior, Interspectives CISV, 73-77.

FERnÁndez-UlloA, Teresa (2004): «Espanglish y cambio de código en el Valle de San Joaquín, California», Symposium Proceedings. <www.csub.edu/ tfernandez ulloa/spanglish.pdf>, 82-92.

Gómez CAPUz, Jean (1997): «Towards a tipological classification of linguistic borrowing (illustrated with anglicisms in Romance languages)", Revista Alicantina de Estudios Ingleses 10, 81-94.

Guest, Greg, Emily Namcy y Marlyn Mitchell (2013): Collecting Qualitative Data, A field Manual for Applied Research, London: SAGE Publications. 
GuMPERZ, Jean (1982): Discourse strategies, Cambridge: Cambridge University Press. HAMERS, Jamers y Michel BlanC (2000): Bilinguality and Bilingualism, Cambridge: Cambridge University Press.

IBARRA, Orreaga (2010): «Alternancia lingüística en el euskera coloquial de los jóvenes vascoparlantes navarros: algunas razones para su uso». En Manuel García et al. (eds.), Interaxió comunicativa i ensenyment de llengües, GIEL (Grupo de Interacción y enseñanza de Lenguas), 249-256.

- (2011): Nafarroako gazteen ahozko diskurtsoa, Universidad Pública de Navarra.

- (2014): «Tipología y pragmática del Code Switching vasco-castellano en el habla informal de jóvenes bilingües», Lapurdum 18, 23-40.

LANTTO, Hanna (2012): «Code-switching, swearing and slang: The colloquial register of Basque in Greater Bilbao», International Journal of bilingualism 18/6, 633-648. <http://ijb.sagepub.com/>.

LóPEZ Morales, Humberto (1994): Sociolingüistica, Madrid: Gredos.

MARTínez, Erika y Mónica SANAPhre (2017): «Transferencia lingüística y alternancia de código en expresiones de manejo de grupo en clases tipo AICLE», Estudios de Lingüistica Aplicada año 35, n. ${ }^{\circ}$ 65, 83-102.

Mendieta, Eva (1999): El préstamo en el español de los Estados Unidos, New York: Peter Lang.

MondADA, Lorenza (2007): «Le code-switching comme ressource pour l'organisation de la parole-en-interaction», Journal of Language Contact, Brill Online Books and Journals edition 1, 168-197.

MuÑOA, Inma (1997): «Pragmatic functions of code-switching among BasqueSpanish bilinguals». En Actas do I Simposio Internacional sobre o bilingüismo, Vigo: University of Vigo, 528-541.

Muysken, Peter (2000): Bilingual Speech: A typology of Code-Mixing, Cambridge: Cambridge University Press.

POPLACK, Shana (1980): «Sometimes I'll start a sentence in Spanish y termino en español: towards a typology of code-switching», Linguistics 18/7-8, 581-618.

RoMAINE, Susanne, (1989): Bilingualism, Oxford: Basil Blackwell.

- (1999): «Bilingual language development». En M. Barrett (ed.), The Development of Language, Sussex: Psychology Press Ltd., 251-275.

WANG, Li-Chien y Hyun Eunsook (2009): "A Study of Sociolinguistic Characteristics of Taiwan Children's Peer-talk in a Mandarin-English-speaking Preschool», Journal of Early Childhood Research 7, 3-26.

ZimMERMANN, Klaus (2002): «La variedad juvenil y la interacción verbal entre jóvenes». En Félix Rodríguez González (ed.), El lenguaje de los jóvenes, Barcelona: Ariel, 195-240. 